U.S. Department of the Interior

U.S. Geological Survey

\title{
Digital aeromagnetic anomaly data from north-east Nicaragua \\ Departamentos Jinotega and Zelaya \\ Nicaragua
}

by

Herbert A. Pierce ${ }^{1}$

Open-File Report OF 94-280

This report is preliminary and has not been reviewed for conformity with U.S. Geological Survey editorial standards or with the North American Stratigraphic Code. Any use of trade, product, or firm names is for descriptive purposes only and does not imply endorsement by the U.S. Government. Although this program has been used by the U.S. Geological Survey, no warranty, expressed of implied, is made by the USGS as to the accuracy and functioning of the program and related program material, nor shall the fact of distribution constitute any such warranty, and no responsibility is assumed by the USGS in connection therewith.

${ }^{1}$ Center for Inter-American Mineral Investigations, 210 E. 7th Street, Tucson, Arizona 85705 


\section{INTRODUCTION}

The Center for Inter-American Mineral Resource Investigations (CIMRI) supports distribution and analysis of geoscientific and mineral resource related information concerning Latin America. CIMRI staff digitized aeromagnetic data for north-east Nicaragua and portions of the Departamentos of Jinotega and Zelaya as part of a preliminary on-going regional assessment of gold in Central America. The data were digitized from eight 1:50,000 scale aeromagnetic contour maps and merged into a single digital data set. The data were used to examine the Chortis/Cholutuega tectonic boundary as well as explore ideas concerning the mineral deposits within the area. This Open-File report, consisting of this text and two (2) 3.5" IBM-PC compatible ASCII magnetic disks, makes the digital data available to the public. Information regarding the source of data and subsequent processing is included below.

In 1964 an aeromagnetic survey funded by the United Nations Special Fund was flown for the Nicaraguan Government by Hunting Survey Corporation Limited, Toronto, Canada. Matthews (1976) published the data as a contour map that covers north-east Nicaragua and extends from about $14^{\circ} 15^{\prime} \mathrm{N}$ to $13^{\circ} 30^{\prime} \mathrm{N}$ latitude and from $85^{\circ} 10^{\prime} \mathrm{W}$ to $83^{\circ} 35^{\prime} \mathrm{W}$ longitude. The contour maps were digitized at points where the magnetic contours intersect the east-west flight lines. The data files include an XYZ ASCII file, three ASCII grids, and three binary "standard USGS" grids; that is, in addition to the XYZ file, there are two of each of the bulleted items listed below:

- raw data merged grid

- residual or International Geomagnetic Reference Field (IGRF) removed grid

- 2nd order residual grid (IGRF removed and a second order surface removed)

These seven data files were transferred to $3.5^{\prime \prime} 1.44 \mathrm{Mbyte}$ disks, readable on IBM-compatible personal computers and are available from the Department of Commerce National Geophysical Data Center ${ }^{1}$.

\section{NATURE AND CONTENTS OF THE 3.5" DISKS}

The data files are backed-up and compressed. Files are backed-up using MS-DOS 6.2 MSBACKUP command and must be restored using the same command. Once the files are restored they must be uncompressed by typing "ARJ E NICARAU". The compression-extraction program ARJ is included with the disks. The user will need 9 MegaBytes of hard disk space for the data files, source code files, and ARJ files after restoring and compressing the files from the two 3.5" diskettes.

Mean ground clearance of the flight lines is 150 meters, mean flight line spacing is 500 meters, and mean control line spacing is 40 kilometers. The aeromagnetic grids have a 500 meter grid interval. These data were presented at the McKelvey Forum in Tucson, Arizona (Pierce, 1994). For most purposes, these grids are the only data files a user will need. FORTRAN 77 code of

1 Address: National Geophysical Data Center, NOAA, Code E/GC1, 325 Broadway, Boulder, CO 80303 U.S.A., Phone 303-497-6826, Fax 303-497-6513. 
selected computer programs that can read most of the above-mentioned files are also included on disk.

All grids are in Universal Transverse Mercator (UTM) coordinates, the central meridian is -85 degrees (minus sign indicates west longitude), and the base latitude is 0 degrees. Units are in kilometers with no false easting or northing. There are a total of 11 data and source code files on the disks; after they are restored and uncompressed the user should have a grand total of 14 files (count includes the restored compressed file, ARJ program, and ARJ help file).

\section{File 1. NICXYZ.ASC:}

The file NICXYZ.ASC is the ASCII version of the XYZ point data, hand-digitized along flight lines by the U.S. Geological Survey. The XYZ ASCII file is in the form of (3F16.6) longitude, latitude, and magnetic field. Latitude and longitude are in decimal degrees and the magnetic field is in gammas (nT).

\section{File 2. NICRAW.ASC:}

The file NICRAW.ASC is the ASCII version of the USGS standard grid file for the north-east Nicaragua area containing the raw magnetic field data from the 1964 Hunting Survey Corporation Limited survey. When converted to binary using program ASCII2SF and examined using program ID, the following output describes the grid:

1) Enter input file name:

2) * UN Aeromag for NE Nicaragua

3) prigrd

4) iproj $=2$ central merid. $=-85.000000$ base lat. $=.000000$

5) $\operatorname{ncol}=303 \quad$ nrow $=172 \quad \mathrm{nz}=1$

6) $\mathrm{x} 0=-4.989051 \mathrm{dx}=.5000000 \mathrm{y} 0=1490.303 \mathrm{dy}=.5000000$

7) Stop - Program terminated.

where:

line 1 = command line

line 2 = file title

line 3 = name of the program used to generate file

line 4 = projection No., central meridian, and base latitude

line $5=$ number of columns, number of rows, and number of $\mathbf{z}$ 
line $6=x$-origin, $x$-spacing, $y$-origin, and $y$-spacing (in $\mathrm{km}$ )

line 7 = program termination

File 3. NICIGRF.ASC:

ASCII version of USGS standard grid file containing residual magnetic field (IGRF for 1964 removed) data from the 1964 Hunting Survey Corporation Limited survey. When converted to binary using program ASCII2SF and examined using program ID, the following output describes the grid:

Enter input file name:

* NE Nic -igrf

prjgrd

iproj $=2$ central merid. $=-85.000000$ base lat. $=.000000$

ncol $=303 \quad$ nrow $=172 \quad \mathrm{nz}=1$

$\mathrm{x} 0=-4.989051 \mathrm{dx}=.5000000 \mathrm{y} 0=1490.303 \mathrm{dy}=.5000000$

Stop - Program terminated.

\section{File 4. NICRES.ASC:}

ASCII version of USGS standard merged grid file containing residual magnetic field (IGRF and a second order field removed) of 1964 Hunting Survey Corporation Limited survey. When converted to binary using program ASCII2SF and examined using program ID, the following output describes the grid:

Enter input file name:

* UN Aeromag for NE Nicaragua

prjgrd

iproj $=2$ central merid. $=-85.000000$ base lat. $=.000000$

ncol $=303$ nrow $=172 \quad \mathrm{nz}=1$

$\mathrm{x} 0=-4.989051 \mathrm{dx}=.5000000 \mathrm{y} 0=1490.303 \mathrm{dy}=.5000000$

Stop - Program terminated. 


\section{File 5. NICRAW.GRD:}

Binary version of the USGS standard grid file containing the raw magnetic field.

File 6. NICIGRF.GRD:

Binary version of the USGS standard grid file containing the residual (IGRF for 1964 removed) magnetic field.

File 7. NICRES.GRD:

Binary version of the USGS standard grid file containing the residual (IGRF and a second order residual field removed) magnetic field.

\section{File 8. ASCII2SF.FOR}

FORTRAN program to convert from ASCII version of USGS standard grid to binary version. For specifications of standard grid file, see Appendix 1.

\section{File 9. SF2ASCII.FOR}

FORTRAN program to convert from binary version of USGS standard grid to ASCII version. For specifications of standard grid file, see Appendix 1.

\section{File 10. ID.FOR}

FORTRAN program to print identification, grid parameters, and minimum and maximum of USGS standard grid.

\section{File 11. NENICOF.DOC}

The ASCII text of this written report. 


\section{APPENDIX 1}

Specifications of USGS standard grid (Cordell and others, 1992):

GRID DATA: (Referred to as "standard file" in some of the programs). Gridded input and output to all the programs are in standardized binary grid format, often with the file name suffix ".grd". Currently, the standard grid applies only to real-valued scalar data in rectangular cells. The grid file consists of a header record followed by one record for each row of data. Row 1 is the first row stored. Origin is in the lower left or southwest corner, starting at row 1, column 1. Row numbers increase upward or northward; column numbers increase to the right or eastward.

Header record (23 4-byte words):

id: 56 ASCII characters of identification

(character*56). pgm: 8 ASCII characters identifying creating program (sometimes); character*8. ncol: number of columns (integer). nrow: number of rows (integer). nz: number of words per data element.

Normally $\mathrm{nz}=1$. (integer). $\mathrm{x} 0$ : position of first column of data, in $\mathrm{km}($ real*4).

$\mathrm{dx}$ : delta $\mathrm{x}$, spacing interval of columns, in $\mathrm{km}\left(\mathrm{real}^{* 4}\right)$.

y0: position of first row of data, in $\mathrm{km}$ (real*4).

dy: delta y, spacing interval of rows, in $\mathrm{km}(\mathrm{real} * 4)$.

Data record: Each data record contains one row of scalar, real-valued data. The first word should contain the row coordinate, but in some programs this rule is not applied. In the data of this report, the first column is always "0". Subsequent words contain data: $f(1, j), f(2, j) \ldots f(n c o l, j)$, for the $j$-th row of data. The sort bit of FORTRAN code below is an example of grid input/output:

read or write (..) id,pgm,ncol,nrow, nz, $\mathrm{x} 0, \mathrm{dx}, \mathrm{y} 0, \mathrm{dy}$

do $10 \mathrm{j}=1$, nrow

10 read or write $(.$.$) y, (f(i, j), i=1$, ncol $)$

All grids are rectangular. Areas within the grid containing no data are flagged by dummy values (DVALS), normally $1.0 \mathrm{e}+38$. 


\section{REFERENCES}

Pierce, Herbert A., 1994, Preliminary interpretation of aeromagnetic data in northeastern Nicaragua possible relation between structure and economic mineral deposits: USGS research on mineral resources - 1994 part A., U.S. Geological Survey Circular no. 1103-A, p74.

Cordell, Lindrith, Phillips, J.D., and Godson, R.H., 1992, U.S. Geological Survey Potential-Field Geophysical Software Version 2.0, U.S. Geological Survey Open File Report 92-18.

Matthews, J.E., 1976, Geomagnetic field of the Caribbean Sea: U.S. Geological Survey, Miscellaneous Field Studies Map, MF-742, 1:2,500,000 scale. 